\title{
Evaluation of the Potency, Neutralizing Antibody Response, and Stability of a Recombinant Fusion Protein Vaccine for Streptococcus pyogenes
}

\author{
E. Burlet, ${ }^{1}$ H. HogenEsch, ${ }^{2}$ A. Dunham, ${ }^{2}$ and G. Morefield ${ }^{1,3}$
}

Received 4 January 2017; accepted 25 February 2017; published online 10 March 2017

\begin{abstract}
Streptococcus pyogenes or group A streptococcus (GAS) is a Gram-positive bacterium that can cause a wide range of diseases, including pharyngitis, impetigo, scarlet fever, necrotizing fasciitis, rheumatic fever, and streptococcal toxic shock syndrome. Despite the increasing burden on global health caused by GAS, there is currently no licensed vaccine available. In this study, we evaluated immunogenicity, induction of neutralizing antibodies, and stability of a new recombinant fusion protein vaccine that targets infections from GAS. The recombinant fusion protein (SpeAB) combines inactive mutant forms of streptococcal pyrogenic exotoxin A (SpeA) and streptococcal pyrogenic exotoxin B (SpeB). The SpeAB vaccine evaluated in this study was adsorbed to an aluminum adjuvant and demonstrated robust immunogenicity, eliciting production of specific neutralizing antibodies against SpeA and SpeB, two major virulence factors of $S$. pyogenes. Stability studies suggest that the vaccine will retain immunogenicity for at least 2 years when stored at refrigerated temperatures. This novel vaccine shows great potential to provide protection against GAS infections and to reduce the burden of GAS disease globally.
\end{abstract}

KEY WORDS: Streptococcus pyogenes; vaccine formulation efficacy.

\section{INTRODUCTION}

Streptococcus pyogenes is a Gram-positive bacterium that causes a wide variety of diseases, from mild infections such as pharyngitis and impetigo to life-threatening diseases, including necrotizing fasciitis, streptococcal toxic shock syndrome, and sequelae such as rheumatic heart disease. These disease states are generally referred to as group A streptococcal (GAS) diseases. Mortality from GAS disease is a global issue with an estimated 500,000 deaths attributed to S. pyogenes infection annually (1). In the USA, there are an estimated 10 million cases of non-invasive disease and between 9000 and 11,500 cases of invasive disease each year. The greatest burden is due to rheumatic heart disease, with a prevalence of at least 15.6 million cases, with 282,000 new cases and 233,000 deaths each year (1). In general, GAS infection is treated with a course of antibiotics; however, antibiotic treatment does not guarantee prevention of complications associated with infection. Additionally, the emergence of antibiotic-resistant strains stresses the need for a safe and effective vaccine for the prevention of GAS disease (2-5). The World Health Organization included GAS vaccine

\footnotetext{
${ }^{1}$ VaxForm, LLC, Bethlehem, Pennsylvania, USA.

${ }^{2}$ Department of Comparative Pathobiology, Purdue University, West Lafayette, Indiana, USA.

${ }^{3}$ To whom correspondence should be addressed. (e-mail: garry.morefield@vaxform.com)
}

development as one of the priorities in the 2014 Global Vaccine Action Plan (6). Vaccines to prevent S. pyogenes infections have been pursued for decades, but their development faces several hurdles. A major concern is that $S$. pyogenes vaccine antigens may contain autoimmune epitopes that could trigger rheumatic heart disease (7). Another hurdle is the complexity of the epidemiology of $S$. pyogenes infections, including the existence of a large number of strains, the wide variety of diseases the bacteria can cause, and the different sites of infection (6). Different strains of GAS are identified by the expression of the antigenically variable $M$ surface protein, a potential vaccine target. The most common genotype is $M_{1}$, but it is desirable to develop a vaccine that is protective across all different genotypes. A 26 -valent and a 30 -valent vaccine have been evaluated and tested in clinical trials $(8,9)$. However, there are more than 150 GAS $M$ serotypes, and their relative frequency can change rapidly. Moreover, epidemiologic surveys suggest that the 26-valent vaccine would provide good coverage of circulating strains of GAS in high-income countries but poor or modest coverage in Africa, the Pacific, Asia, and the Middle East (10). More importantly, crossreactivity with $M$ protein has been implicated in the pathogenesis of rheumatic heart disease raising safety concerns about $M$ protein-based vaccines (11). So far, all GAStargeting vaccines tested in clinical trials are based on conserved $M$ proteins (12). We have chosen an alternative strategy by developing a vaccine based on non- $M$ proteins 
that are conserved among GAS strains. The recombinant fusion protein antigen (SpeAB) was engineered combining streptococcal pyrogenic exotoxin A (SpeA) with streptococcal pyrogenic exotoxin B (SpeB) from S. pyogenes (13). SpeA is a superantigen which enables bacteria to evade immune responses by disabling recognition by adaptive immunity (14). SpeA cross-links major histocompatibility complex class II molecules with $\mathrm{T}$ cell receptors resulting in polyclonal activation of $\mathrm{T}$ cells. The systemically increased levels of inflammatory cytokines can induce toxic shock (15). The SpeA utilized in the fusion protein antigen was genetically mutated at the leucine located at position 42 inactivating superantigen activity (13). SpeB is an extracellular cysteine protease that is secreted by most $S$. pyogenes strains (16). SpeB can cleave or degrade host serum proteins such as human extracellular matrix, immunoglobulins, complement components, and even GAS surface and secreted proteins (17). SpeB is not homologous in structure to SpeA and has no superantigen functionality but is a key virulence factor in GAS pathogenesis. Analysis of sera obtained from patients with invasive GAS infection has demonstrated SpeB-specific antibody production (18). SpeB utilized in the fusion protein antigen was genetically mutated at the cysteine located at position 47 , inactivating the enzyme. Immunization with the SpeAB fusion protein protected HLA-DQ8 transgenic mice against a challenge with wild-type SpeA (13). Following the fusion protein discovery and efficacy, a rational, scientific approach to vaccine development was utilized in our laboratory to determine the optimal formulation conditions and the optimal aluminum adjuvant type (19). In the current study, the stability and potency of the vaccine were tested with various adjuvant doses and heat-induced degraded vaccines in vivo including the ability to induce SpeA- and SpeBneutralizing antibodies, and the shelf life of the vaccine was determined. This novel vaccine shows great potential to protect against GAS infections and reduce the burden of GAS disease globally.

\section{MATERIAL AND METHODS}

\section{Vaccine Preparation}

SpeAB recombinant protein: The cloning of SpeAB DNA into the vector pT7Blue (Novagen) was performed as described (13). Fermentation and purification of the fusion recombinant protein were conducted at VaxForm. Briefly, kanamycin-resistant Escherichia coli BL21(DE3) cells were cultured overnight at $37^{\circ} \mathrm{C}$ in $25 \mathrm{~g} / \mathrm{L}$ Lennox Broth (LB) and $50 \mu \mathrm{g} / \mathrm{mL}$ kanamycin. The next day, $1 \mathrm{~L}$ of broth was prewarmed to $37^{\circ} \mathrm{C}$, and $20 \mathrm{~mL}$ of the overnight culture was transferred to the $1-\mathrm{L}$ vessel. The bacteria were then grown to an OD of 0.400 for $2 \mathrm{~h}$. IPTG was added at $1 \mathrm{mM}$ and the temperature of the culture was reduced to $30^{\circ} \mathrm{C}$ and incubated overnight. The next day, the bacteria were harvested for purification. The fermentation broth was exchanged with Tris-buffered saline (TBS) using a 1000-kD filter and tangential flow filtration (TFF) system (EMD Millipore). Cells were lysed with lysozyme, surfactants, and nuclease. The released inclusion bodies were then washed with TBS and dissolved in $6 \mathrm{M}$ urea overnight at room temperature. The SpeAB was then refolded into Tris-Tween buffer using a $30-\mathrm{kD}$ filter on the TFF. Endotoxin was removed by addition of sodium deoxycholate followed by purification into Tris-Tween buffer with a $30-\mathrm{kD}$ filter. Finally, sucrose was added to $10 \% \mathrm{w} / \mathrm{v}$ and the bulk was sterile filtered and stored refrigerated.

Formulation of the vaccine: Vaccines were prepared by mixing appropriate amount of SpeAB protein with phosphate-treated "Alhydrogel 85" (Brenntag, Denmark) and buffer to obtain $50 \mu \mathrm{g} / \mathrm{mL}$ SpeAB, 1.7, 0.85 , or $0.425 \mathrm{mg} / \mathrm{mL}$ of aluminum (Al ion), in $20 \mathrm{mM}$ Tris (EMD Millipore, Germany) buffer with $10 \%$ sucrose (EMD Millipore) and $0.02 \%$ polysorbate 20 (Amresco, Solon, $\mathrm{OH}$ ). All vaccines had $80 \%$ or more antigen adsorbed to the adjuvant. The $\mathrm{pH}$ was $7.5-8.5$ as previous experiments had demonstrated that SpeAB is most stable in this range (19). Control groups contained either phosphate-treated Alhydrogel (AHP) only or $50 \mu \mathrm{g} / \mathrm{mL}$ of SpeAB only, both in Tris buffer with sucrose and polysorbate 20 .

\section{Mouse Studies}

Adjuvant dose-response and antigen-forced desorption immunogenicity studies of the SpeAB vaccine were conducted in 7-week-old female BALB/c mice (Envigo, Indianapolis, IN). The experiments were approved by the Purdue University Animal Care and Use Committee. Three doses of aluminum adjuvant were tested $(0.425,0.85$, or $1.7 \mathrm{mg} / \mathrm{mL})$ with at least $80 \%$ of the antigen adsorbed to aluminum adjuvant. Three adsorption states of SpeAB antigen $(100 \%$ adsorbed, 30 and $60 \%$ desorbed) were used to evaluate the correlation between the vaccine potency and its stability. SpeAB antigen was incubated at $45^{\circ} \mathrm{C}$ for 6 or 14 days to obtain 30 and $60 \%$ desorption of the antigen, respectively (see stability methods for details on adsorption measurement). In both studies, mice were immunized (10 per group) with $0.1 \mathrm{~mL}$ vaccine intramuscularly on days 0 and 14 of the study. The injected dose of SpeAB was therefore $5 \mu \mathrm{g}$, while the amount of aluminum injected was $0.85,0.425$, or $0.212 \mathrm{mg}$. Sera were collected on days 14 and 28 to evaluate SpeAB titers by ELISA. Briefly, wells were coated with $10 \mu \mathrm{g} / \mathrm{mL}$ SpeAB overnight, blocked with 1\% BSA (VWR, Radnor, PA) in PBS-Tween 20 buffer, and incubated for $2 \mathrm{~h}$ with 1 : 5000 diluted serum samples. Peroxidase-labeled anti-mouse IgG (Sigma, St. Louis, MO) was added followed by $3,3^{\prime}, 5,5^{\prime}-$ tetramethylbenzidine (TMB; Sigma). Absorbance was determined at $450 \mathrm{~nm}$ (Molecular Devices M2e).

\section{SpeA and SpeB Neutralization Assays}

SpeA neutralization by SpeAB vaccine-elicited antibodies was evaluated in human peripheral blood mononuclear cells (PBMCs; ZenBio, Research Triangle Park, NC) by the antibodies' ability to inhibit SpeA-induced polyclonal activation. Briefly, PBMCs were grown in $10 \%$ human serum at $37^{\circ} \mathrm{C}$, $5 \% \mathrm{CO}_{2}$. Mouse sera and recombinant SpeA (Toxin Tech, Saratosa, FL) were mixed at $37^{\circ} \mathrm{C}$ for $1 \mathrm{~h}$. Sera and SpeA mix were then added to PBMCs and incubated for 4 days. Alamar Blue dye (Thermo Fischer), an oxidation-reduction indicator used to monitor cell proliferation, was then added to the cells (10\% of the total volume). PBMC proliferation rate was quantified by measuring percent reduction of Alamar Blue 
dye at absorbance $570 \mathrm{~nm}$ and reference at $600 \mathrm{~nm}$ (Molecular Devices M2e).

SpeB neutralization was assessed by monitoring the SpeB protease activity on a highly quenched protein substrate in presence of vaccine-elicited antibodies. Fluoro Protease Assay Kit (G Biosciences, St. Louis, MO), an assay that uses fluorescein isothiocyanate (FITC)-labeled casein as a general protease substrate, was used. The fluorescein label on the FITC casein is highly quenched. When the proteases present in the sample of interest digest the FITC casein substrate into smaller peptides, the quenching of the fluorescence label is released and the fluorescence of the substrate is increased. The fluorescence of the FITC-labeled peptide is measured with excitation at $485 \mathrm{~nm}$ and emission at $530 \mathrm{~nm}$ to determine protease activity. Wild-type SpeB (Toxin Technology, Sarasota, FL) was first incubated with $5 \% \beta$ mercaptoethanol (VWR) at $37^{\circ} \mathrm{C}$ to activate the protein. Reduced SpeB was mixed with immunized-mouse sera (from mice immunized with SpeAB vaccine $v s$ mice immunized with adjuvant only) and incubated at $37^{\circ} \mathrm{C}$ for $1 \mathrm{~h}$. The mixture was then exposed to FITC casein substrate, and the kinetic activity of SpeB was quantified measuring fluorescence.

\section{Stability Studies}

The stability of the SpeAB vaccine was evaluated in accelerated conditions (temperatures of 37 and $45^{\circ} \mathrm{C}$ ) and refrigerated temperature $\left(2-8^{\circ} \mathrm{C}\right)$. For each time point (every 3 months), three vials were used for analysis. $\mathrm{pH}$, adsorption assays, and ELISA were performed on the vaccine to monitor its stability. The $\mathrm{pH}$ of the whole liquid suspension was measured using a sympHony $\mathrm{pH}$ meter.

For adsorption assays, $100 \mu \mathrm{L}$ of the vaccine was centrifuged (Eppendorf 5424/5424R microcentrifuge) at $10,000 \mathrm{~g}$ for $5 \mathrm{~min}$. The supernatant was collected and total protein assay (Thermo Scientific Pierce BCA Protein Assay Kit) was performed. Bovine serum albumin (BSA) standards provided in the kit were used as standards. The pre-diluted samples were further diluted in the vaccine buffer (Tris sucrose-Tween) to obtain a standard curve ranging from 3 to $100 \mu \mathrm{g} / \mathrm{mL}$. To determine the percentage of SpeAB adsorbed to the adjuvant, the protein that was detected in the supernatant was subtracted from $50 \mu \mathrm{g} / \mathrm{mL}$, the concentration of SpeAB in the vaccine. That number was divided by 50 and then multiplied by 100 to obtain the percentage.

Sandwich ELISA was performed on the desorbed antigen to evaluate the vaccine antigenicity as aluminum would interfere with the assay. Of the vaccine, $150 \mu \mathrm{L}$ was centrifuged at $10,000 \mathrm{rcf}$ for $5 \mathrm{~min}$. The supernatant was removed. Antigen was then desorbed from aluminum adjuvant by incubating the aluminum pellet in desorption buffer ( $75 \mathrm{mM} \mathrm{PO}_{4}, 150 \mathrm{mM} \mathrm{NaCl}, 0.01 \%$ Triton-X 100) at $37^{\circ} \mathrm{C}$ for $24 \mathrm{~h}$. The next day, the samples were centrifuged at 10,000 rcf for $5 \mathrm{~min}$. Supernatants were collected and further analyzed by ELISA. Each well of a microplate was coated with $100 \mu \mathrm{L}$ of anti-SpeA antibody (Abcam, Cambridge, MA) diluted 1:1000 in $\mathrm{PBS}$, incubated at $37^{\circ} \mathrm{C}$ for an hour. Plate was washed with PBS- $0.02 \%$ Tween 20 three times. Of the blocking buffer (1\% BSA-PBS), $100 \mu \mathrm{L}$ was added to the wells. Every step was performed at $37^{\circ} \mathrm{C}$ for $1 \mathrm{~h}$ with $100 \mu \mathrm{L}$ of reagent or sample, followed by thorough washing of the wells. Samples and standard (SpeAB antigen control formulated without AHP1) were added to the plate. Anti-SpeB antibody (Abcam, Cambridge, MA) 1:5000 dilution was then added, followed by anti-rabbit HRP-conjugated (1:80,000 dilution). TMB substrate (KPL Inc., Maryland) was added to each well and was incubated at room temperature, protected from light for $30 \mathrm{~min}$. Reaction was stopped with $3 \mathrm{M} \mathrm{H}_{2} \mathrm{SO}_{4}$. Absorbance was read at $450 \mathrm{~nm}$. Concentrations determined by ELISA were used for the Arrhenius plot.

In the stability assays, SpeAB antigen control stored at $-20^{\circ} \mathrm{C}$ was used as a reference. Its concentration was measured in the adsorption assays by BCA assay to determine the maximum SpeAB concentration and percentage adsorbed, and it was serially diluted and used for the ELISA standard curve.

\section{Statistical Analysis}

Data are presented as the mean \pm SEM for experimental groups. Normally distributed data were analyzed for differences among experimental groups by one-way ANOVA. * represents $p$ value $<0.05$, ** represents $p$ value $<0.01$, and $* * *$ represents $p$ value $<0.005$.

\section{RESULTS}

To determine the optimal adjuvant dose needed for the vaccine, a dose-response potency study was performed in $\mathrm{BALB} / \mathrm{c}$ mice. The highest dose level of aluminum currently approved by regulatory authorities for human use is $1.7 \mathrm{mg}$ $\mathrm{Al} / \mathrm{mL}$ - therefore, this dose was selected as the upper limit dose in the animal study. BALB/c mice were administered Spe AB vaccine formulated with $0,0.425,0.85$, or $1.7 \mathrm{mg} \mathrm{Al} /$ $\mathrm{mL}$ on days 0 and 21. Mice immunized with 0.85 and $1.7 \mathrm{mg} /$ $\mathrm{mL}$ of adjuvant had a higher $\mathrm{IgG}$ titer compared with mice immunized with antigen alone (Fig. 1a). However, there was no significant difference between the $0.425-, 0.85-$, and $1.7-$ $\mathrm{mg} / \mathrm{mL}$ aluminum-dose groups.

Previous studies in our laboratory demonstrated that adsorbed SpeAB induces a stronger immune response compared with non-adsorbed SpeAB (19). The impact of heat-induced desorption of an adsorbed formulation following storage under accelerated conditions on potency was unknown. In this study, forced desorption was performed by incubating the vaccine at $45^{\circ} \mathrm{C}$ for 6 and 14 days, resulting in 30 and $60 \%$ desorption of the antigen, respectively, to evaluate the correlation between the vaccine stability and its potency. There was no significant difference in $\mathrm{IgG}$ titers in mice immunized with the $100 \%$ adsorbed, $30 \%$ desorbed, or $60 \%$ desorbed vaccine (Fig. 1b); however, there was a trend towards lower antibody titer as desorption increases.

Two neutralization assays were developed for each of the virulence factors of the fusion protein, SpeA and SpeB, to evaluate the efficacy of the vaccine in vitro. The presence of neutralizing antibodies specific to SpeA, a superantigen, was evaluated by their ability to inhibit SpeA-induced polyclonal activation of human PBMCs. Figure 2 shows that the addition of SpeA induced PBMC proliferation, which was inhibited when serum from SpeAB-vaccinated mice was added to the cells. The PBMC proliferation was not inhibited with serum from adjuvant control-vaccinated mice. 
a

Effect of adjuvant dose on the antibody titer

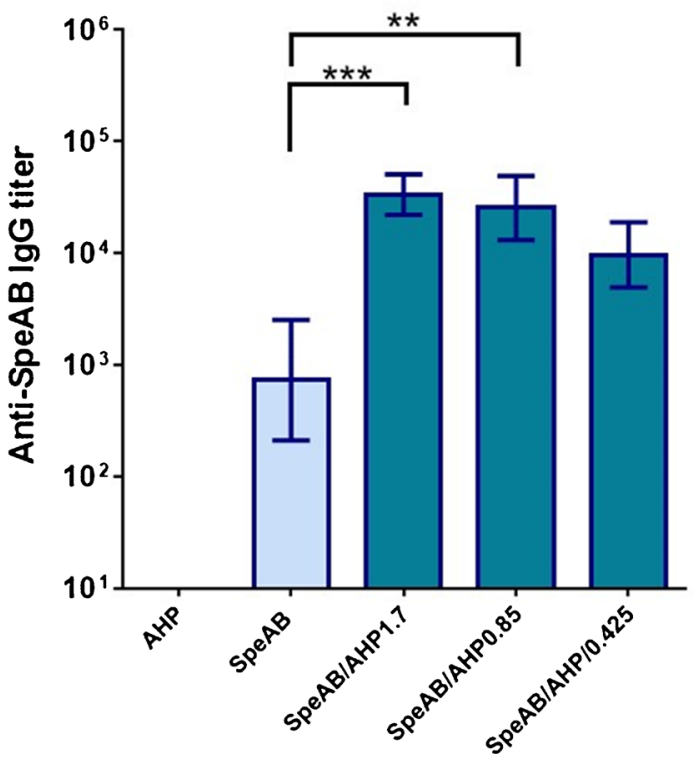

b

Effect of antigen desorption on antibody titer

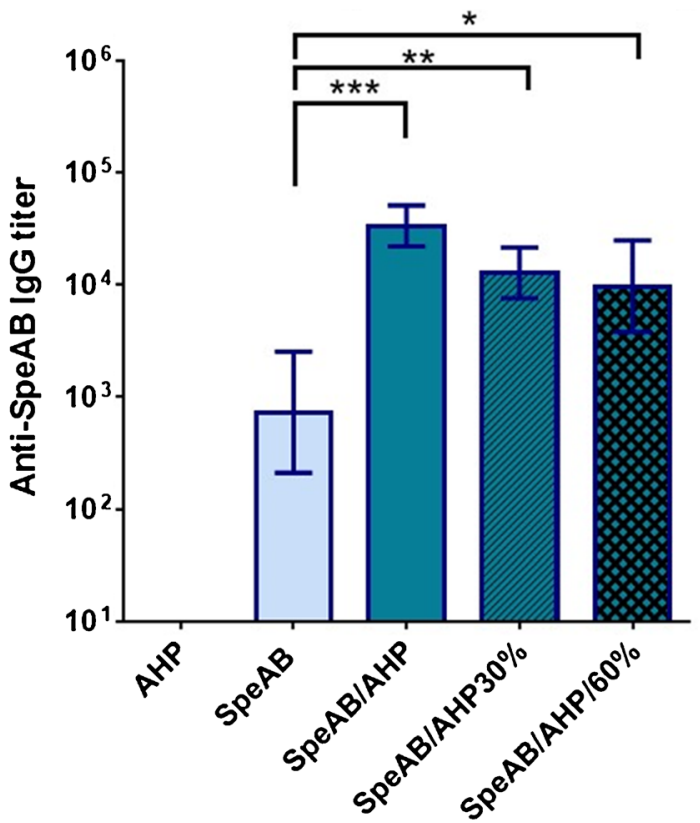

Fig. 1. Potency study of SpeAB vaccine in BALB/c mice. Adjuvant and antigen dose-response immunogenicity studies of the SpeAB vaccine were conducted in 7-week-old female BALB/c mice. Of the SpeAB, $50 \mu \mathrm{g} / \mathrm{mL}$ was combined with 0 , $0.425,0.85$, or $1.7 \mathrm{mg} / \mathrm{mL}$ of aluminum adjuvant for the adjuvant-dose study (a), with at least $80 \%$ of the antigen adsorbed. Three adsorption states of the SpeAB antigen (100\% adsorbed, $30 \%$ desorbed, and $60 \%$ desorbed; see methods for details) in $1.7 \mathrm{mg} / \mathrm{mL}$ of adjuvant were used to evaluate the correlation between the vaccine stability and its potency (b). In both studies, mice were immunized (10 per group) with $0.1 \mathrm{~mL}$ vaccine IM on days 0 and 14 of the study. Sera were collected on days 14 and 28 to evaluate SpeAB titers by ELISA. $* p<0.05, * * p<0.01, * * * p<0.005$

The presence of neutralizing antibodies specific to SpeB, a cysteine protease, was assessed using the Fluoro Protease Assay Kit. Serum samples from mice immunized with SpeAB vaccine significantly decreased SpeB activity when combined with FITC-labeled casein (both at 5 and

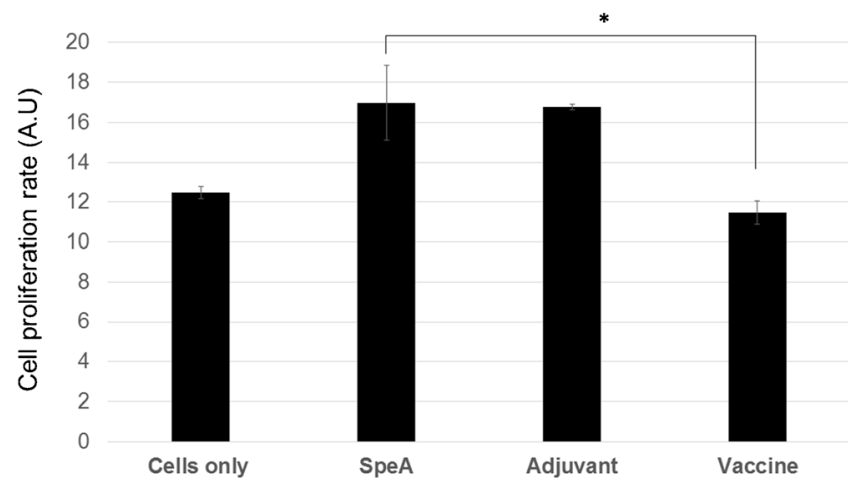

Fig. 2. Effect of vaccine-elicited antibodies on SpeA activity. SpeA neutralization by Spe AB vaccine-elicited antibodies was evaluated in human peripheral blood mononuclear cells (PBMCs). Sera from mice immunized with either $50 \mu \mathrm{g} / \mathrm{mL}$ SpeAB/1.7 mg/mL Al or $1.7 \mathrm{mg} / \mathrm{mL}$ $\mathrm{Al}$ only were mixed with recombinant SpeA at $37^{\circ} \mathrm{C}$ for $1 \mathrm{~h}$. The mix was then added to PBMCs and incubated for 4 days. Alamar Blue dye was then added to the cells (10\% of the total volume). PBMC proliferation rate was quantified by measuring percent reduction of Alamar Blue dye at absorbance $570 \mathrm{~nm}$ and reference at $600 \mathrm{~nm}$. Bars represent the mean of $n=3$ experiments \pm SE (AU stands for arbitrary units). $* p<0.05$
$10 \mathrm{ng} / \mu \mathrm{L})$ compared to sera from mice immunized with adjuvant alone (Fig. 3).

In addition to the ability to induce functional neutralizing antibodies, an effective vaccine should demonstrate good stability over time. The $\mathrm{pH}$ of the vaccine is important to monitor as a change in $\mathrm{pH}$ can impact the structural stability of the antigen and its interaction with the aluminum adjuvant, resulting in decreased immunogenicity of the vaccine. Previous experiments in our laboratory (19) showed that the vaccine is most stable near $\mathrm{pH}$ 8.0. The $\mathrm{pH}$ of vaccines stored at $2-8^{\circ} \mathrm{C}$ was measured every 3 months over 24 months and showed strong stability with the $\mathrm{pH}$ remaining between 8.0 and 8.1 (data not shown). Another important factor that may affect the SpeAB vaccine potency is antigen adsorption to the aluminum adjuvant. We reported previously (19) that the vaccine is most potent when the majority of SpeAB is adsorbed to the aluminum adjuvant ( $80 \%$ or above). Adsorption was monitored every 3 months over a 24-month period (Fig. 4). The adsorption of SpeAB increased in the first 3 months from $80 \%$ to reach an equilibrium, close to $100 \%$ adsorption. While the percentage adsorption fluctuated slightly, it remained above $90 \%$ over the 24 months of storage, demonstrating robust stability.

The vaccine antigenicity was evaluated by ELISA in accelerated and real-time stability studies to obtain data for expiration dating and temperature excursion evaluation of the vaccine. Arrhenius kinetics were used to evaluate the stability of the antigen $v s$ temperature (Fig. 5). If a plot of $\ln (k)$ vs $1 / T$ is linear, the data obtained from the accelerated conditions can be extrapolated to real-time storage conditions 


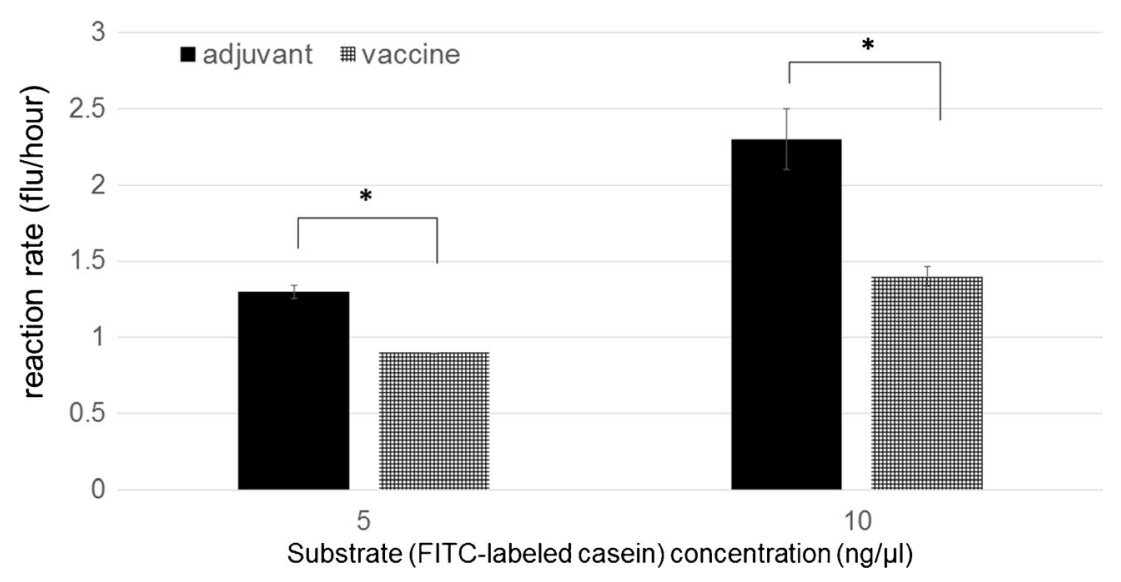

Fig. 3. Effect of vaccine-elicited antibodies on SpeB activity. SpeB neutralization by Spe $A B$ vaccine-elicited antibodies was evaluated in vitro by monitoring the SpeB protease activity using highly quenched FITC casein protein as a substrate. Wild-type SpeB was first incubated with $5 \% \beta$-mercaptoethanol at $37^{\circ} \mathrm{C}$ to activate the enzyme. Reduced SpeB was then mixed with sera (from mice immunized with $50 \mu \mathrm{g} / \mathrm{mL}$ SpeAB and $1.7 \mathrm{mg} / \mathrm{mL} \mathrm{Al} v s$ mice immunized with $1.7 \mathrm{mg} / \mathrm{mL} \mathrm{Al}$ only) and incubated at $37^{\circ} \mathrm{C}$ for $1 \mathrm{~h}$. The mix was then exposed to FITC casein substrate $(5$ or $10 \mathrm{ng} / \mu \mathrm{L}$ ), and the kinetic activity of SpeB was quantified measuring fluorescence at excitation $485 \mathrm{~nm}$ and emission $535 \mathrm{~nm}$. Bars represent the mean of $n=3$ experiments \pm SE. ${ }^{*} p<0.05$

such as refrigerated at $5^{\circ} \mathrm{C}(20)$. In our study, accelerated stability was evaluated at 37 and $45^{\circ} \mathrm{C}$ and exhibited linearity when plotted with $5^{\circ} \mathrm{C}$ stability data (Fig. 5). Arrhenius plot showed perfect linear curve when plotting the logarithm of the rate constant degradation of Spe AB (in $\mu \mathrm{g} / \mathrm{mL}$ as determined by ELISA) against inversed absolute temperature $\left(45,37\right.$, and $\left.5^{\circ} \mathrm{C}\right)$. Using this plot, the half-life of the vaccine can be extrapolated. According to the Arrhenius equation, half-life would be approximately 2.5 years at $2-8^{\circ} \mathrm{C}$.

\section{DISCUSSION}

This study shows that a SpeAB recombinant vaccine formulated with aluminum adjuvant induced SpeA- and SpeB-neutralizing antibodies in mice and demonstrated long-term stability with potential for at least 2-year shelf life

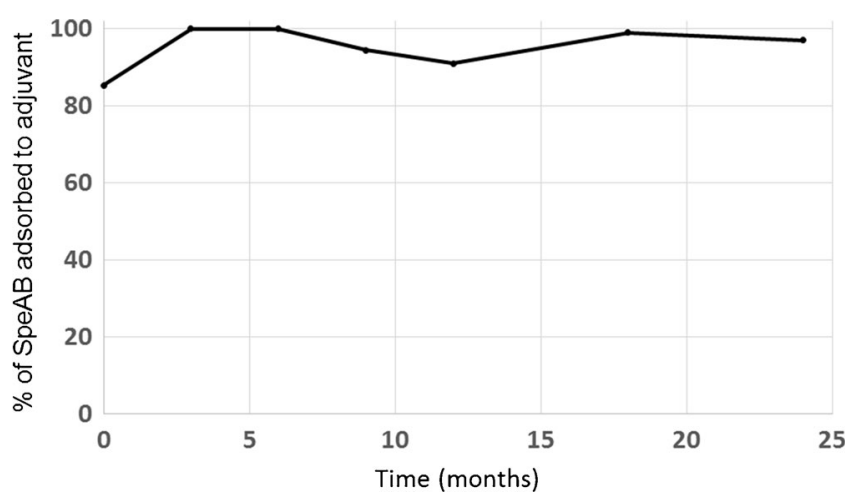

Fig. 4. Spe $A B$ vaccine adsorption stability over time. Spe $A B$ vaccine $(50 \mu \mathrm{g} / \mathrm{mL}, 1 \mathrm{mg} / \mathrm{mL}$ adjuvant, in Tris sucrose-Tween 20 buffer) were stored at $2-8^{\circ} \mathrm{C}$. Three vials were taken out every 3 months to evaluate adsorption. Of the vaccine from each vial, $100 \mu \mathrm{L}$ was centrifuged at 10,000 rcf for $5 \mathrm{~min}$. Supernatant was collected and protein assay (by BCA; see methods for details) was performed on the supernatant at $5^{\circ} \mathrm{C}$. This suggests that the vaccine has excellent potential to prevent GAS-induced disease. The antibody response was improved in the presence of aluminum-containing adjuvant (Fig. 1), confirming our findings from a previous animal study (19). Aluminum-containing adjuvants have been used in vaccines to boost the immune response for more than seven decades (21,22). Despite their wide spread use, the mechanism of action of aluminum-based adjuvants is not fully understood. Some studies demonstrate that maximized adsorption results in optimal immune response, while other studies suggest a decrease in stability in vaccines with high adsorption (23). Consequently, the formulation development for aluminum-containing vaccines is mainly based on empirical trials. Many aspects of aluminum hydroxide-based adjuvant vaccines can influence the immune response, including the strength of the adsorption, dosage of adjuvant, and the nature of antigens (24). All of these factors were taken into consideration during the development of the Spe AB vaccine to ensure the optimal formulation. Adsorbed and non-adsorbed formulations of SpeAB vaccines were evaluated in mice, and results showed that adsorbed formulations exhibited significantly higher immune response than the non-adsorbed formulations (19). In that study, the two different states of adsorption were obtained by treating the aluminum-hydroxide adjuvant with different amount of phosphate to result in more or less electrostatic repulsion. It was found that the higher phosphate treatment resulted in more electrostatic repulsion and a largely desorbed antigen. This non-adsorbed antigen induced a less potent immune response compared to the adsorbed antigen (adjuvant treated with low amount of phosphate). In this study, the adsorbed antigen formulation was used in all vaccines. Heat-induced desorption of the antigen was performed to mimic antigen degradation and evaluate the correlation between stability and potency. Results from the forced-desorption study suggest that the SpeAB vaccine is very robust as there were no significant differences in $\mathrm{IgG}$ titers in mice immunized with 


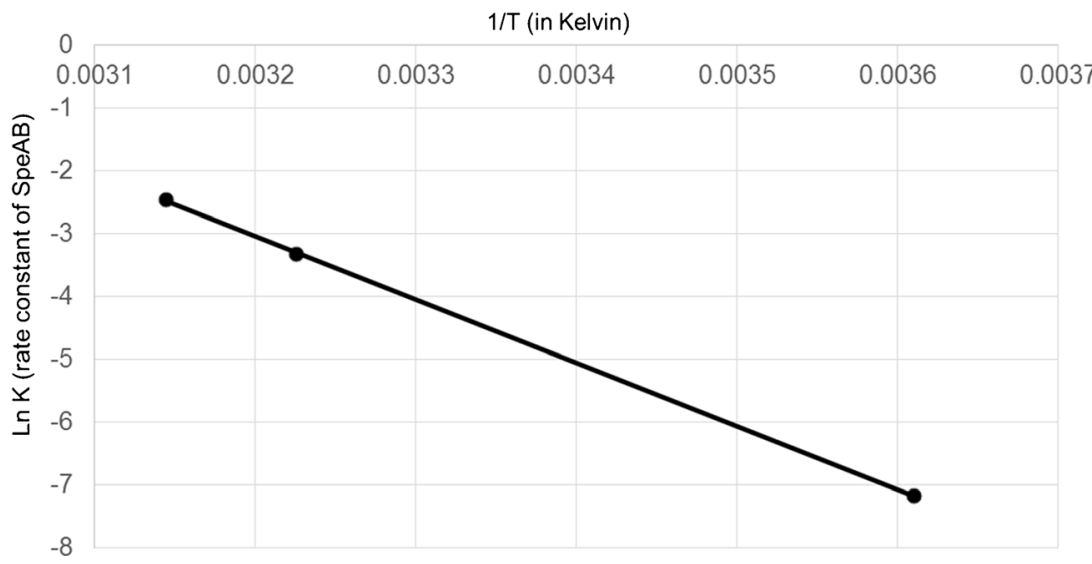

Fig. 5. Arrhenius plot SpeAB rate constant $v s$ temperature. ELISAs (see methods for details) were performed to evaluate the vaccine antigenicity in accelerated and real-time stability studies. Arrhenius kinetics was used to evaluate the rate constant of the antigen in different temperature conditions. The Arrhenius equation is defined by $k=A_{e}^{-(E a / R T)}$ or $\ln (k)=-\left(E_{a} / \mathrm{RT}\right)+\ln A$, where $k$ is the reaction rate constant of the antigen, $A$ is the preexponential factor, $E_{a}$ is the activation energy, $R$ is the gas constant, and $T$ is the temperature in Kelvin. In our studies, 5,37 , and $45^{\circ} \mathrm{C}$, we used to plot $\ln (k)$ vs $1 / T$

full, $30 \%$ desorbed, or $60 \%$ desorbed vaccine. Additionally, the $60 \%$ desorbed vaccine was still significantly more potent than antigen alone, suggesting strong potency of the remaining adsorbed antigen even after exposure to high temperatures. In addition to the in vivo study, the correlation between adsorption state and immunogenicity was evaluated in vitro (Fig. 5). ELISA and adsorption assays were performed on Spe AB vaccine stored at 37 and $45^{\circ} \mathrm{C}$. Pearson's correlation factor between the two trends was 0.92 at $37^{\circ} \mathrm{C}$ and 0.84 at $45^{\circ} \mathrm{C}$, showing significant positive correlation between adsorption and antigenicity. Together, these data show that heat-induced degradation affects the function of the antigen (as seen in ELISA) but does not impact the potency in animals as much. This suggests that the antigen that remains adsorbed to the aluminum is sufficient to induce an immune response and further confirms the robustness of the vaccine. The aluminum dose-effect study confirmed the importance and need of the adjuvant for the SpeAB vaccine potency. There was no significant difference between 0.85 - and $1.7-\mathrm{mg} /$ $\mathrm{mL}$ aluminum-immunized mice. However, there was no significant difference in immunogenicity between antigen alone and $0.425 \mathrm{mg} / \mathrm{mL}$ adjuvant, suggesting that $0.85 \mathrm{mg} /$ $\mathrm{mL}$ is the minimum adjuvant dose needed to significantly enhance immunogenicity. A pre-clinical safety study was conducted shortly after the adjuvant-dose study, where $1 \mathrm{mg} / \mathrm{mL}$ of $\mathrm{Al}$ was used in the Spe AB vaccine (25). The absence of clinical toxicity in two species (including rabbits injected with two full human doses of $0.5 \mathrm{~mL}$ ) confirms the safety of the vaccine using aluminum as an adjuvant.

Results from real-time and accelerated stability studies confirm that SpeAB is a robust vaccine (Fig. 5). The $r^{2}$ value of the Arrhenius plot using $X$-order degradation rate constants from 37,45 , and $5^{\circ} \mathrm{C}$ was 0.999 , demonstrating high correlation between the antigen degradation and temperature. Using this plot, the time required for the antigen to reach 30 and $60 \%$ degradation can be extrapolated. According to the Arrhenius equation, it would take 1.8 years for the SpeAB vaccine to become $30 \%$ degraded and 4.3 years for it to be $60 \%$ degraded. As the potency study suggests (Fig. 1), these degradation states have minimal effect on the potency of the vaccine. The vaccine can remain stable at refrigerated temperatures $\left(2-8^{\circ} \mathrm{C}\right)$ for a minimum of 2 years. Results from neutralization assays demonstrated that the vaccine elicits specific functional antibodies against both SpeA and SpeB. SpeA is a superantigen associated with scarlet fever, toxic shock, and is a major virulence factor for invasive GAS disease (26-28). SpeB is a secreted and cell wall-associated toxin important in the colonization of the host that has been found to be expressed by the majority of S.pyogenes strains (16). The ability of the SpeAB vaccine to elicit protective antibodies against both of these key virulence factors demonstrates the potential for the SpeAB vaccine to induce protective responses against most strains and diseases caused by GAS.

\section{CONCLUSIONS}

Results from the dose-response studies demonstrated that the SpeAB vaccine elicits a strong immune response in mice, according to the IgG titers in mice immunized with reduced adjuvant and partially degraded SpeAB. The vaccine induces neutralizing antibodies that mediate protection against SpeA and SpeB, two major virulence factors expressed by the majority of GAS strains. Results from the stability studies suggest strong antigenicity of the vaccine after 2 years of storage at $2-8^{\circ} \mathrm{C}$. In conclusion, this novel vaccine shows great potential to protect against GAS infections and to reduce the burden of GAS disease globally.

\section{ACKNOWLEDGEMENTS}

This work is supported by the US Army Medical Research and Materiel Command under Contract No. W81XWH-12-C-0183. The views, opinions, and/or findings contained in this report are those of the author(s) and should not be construed as an official Department of the Army position, policy, or decision unless so designated by other documentation. 


\section{COMPLIANCE WITH ETHICAL STANDARDS}

In conducting research using animals, the investigator(s) adhered to the Animal Welfare Act Regulations and other Federal Statutes relating to animals and experiments involving animals and the principles set forth in the current version of the Guide for Care and Use of Laboratory Animals, National Research Council.

\section{REFERENCES}

1. Carapetis JR, Steer AC, Mulholland EK, Weber M. The global burden of group A streptococcal infections. Lancet Infect Dis. 2005;5(11):685-94.

2. Reinert RR, Lutticken R, Al-Lahham A. High-level fluoroquinolone resistance in a clinical Streptoccoccus pyogenes isolate in Germany. Clin Microbiol Infect. 2004;10:659-62.

3. Buxbaum A, Forsthuber S, Sauermann R, Gattringer R, Graninger W, Georgopoulos A. Development of macrolideresistance and comparative activity of telithromycin in streptococci in Austria, 1996-2002. Int J Antimicrob Agents. 2004;24:397-400.

4. Stevens DL. Streptococcal toxic shock syndrome associated with necrotizing fasciitis. Annu Rev Med. 2000;51:271-88.

5. Musser JM, Krause RM. The revival of group A streptococcal diseases with a commentary on staphylococcal toxic shock syndrome. In: Krause RM, Fauci A, editors. Emerging infections. New York: Academic Press; 1998. p. 185-218.

6. Global Vaccine Action Plan. Secretariat Annual Report 2014. http://www.who.int/immunization/global_vaccine_action_plan/ gvap_secretariat_report_2014.pdf?ua=1

7. Massell BF, Honikman LH, Amezcua J. Rheumatic fever following streptococcal vaccination. Report of three cases. J Am Med Assoc. 1969;207:1115.

8. Steer AC, Magor G, Jenney AW, Kado J, Good MF, McMillan $\mathrm{D}$, et al. Emm and C-repeat region molecular typing of betahemolytic streptococci in a tropical country: implications for vaccine development. J Clin Microbiol. 2009a;47(8):2502-9.

9. Dale JB, Penfound TA, Chiang EY, Walton WJ. New 30-valent $M$ protein-based vaccine evokes crossopsonic antibodies against non-vaccine serotypes of group A streptococci. Vaccine. 2011;29(46):8175-8.

10. Steer AC, Carapetis JR, Dale JB, Frase JD, Good MF, Guilherme L, et al. Status of research and development of vaccines for Streptococcus pyogenes. Vaccine. 2016;34:2953-8.

11. Steer AC, Batzloff MR, Mulholland K, Carapetis JR. Group A streptococcal vaccines: 419 facts versus fantasy. Curr Opin Inf Dis. 2009b;22:544-52.

12. Hu MC, Walls MA, Stroop SD, Reddish MA, Beall B, Dale JB. Immunogenicity of a 26-valent group A streptococcal vaccine. Infect Immun. 2002;70(4):2171-7.
13. Ulrich R. Vaccine based on a ubiquitous cysteinyl protease and streptococcal pyrogenic exotoxin A protects against Streptococcus pyogenes sepsis and toxic shock. J Immune Based Ther Vaccine. 2008;6:8.

14. Ulrich RG, Bavari S, Olson M. Bacterial superantigens in human diseases: structure, function and diversity. Trends Microbiol. 1995;3:463-8.

15. Norrby-Teglund A, Pauksens K, Norgren M, Holm SE. Correlation between serum TNF-alpha and IL-6 levels and severity of group A streptococcal infections. Scand J Infect Dis. 1995;27:125-30.

16. Olsen RJ, Raghuram A, Cantu C, Hartman MH, Jimenez FE, Lee $\mathrm{S}$, et al. The majority of 9,729 group A streptococcus strains causing disease secrete SpeB cysteine protease: pathogenesis implications. Infect Immun. 2015;83:4750-8.

17. Chiang-Ni C, Wu JJ. Effects of streptococcal pyrogenic exotoxin B on pathogenesis of Streptococcus pyogenes. J Formos Med Assoc. 2008;107(9):677-85.

18. Akesson P, Rasmussen M, Mascini E, von Pawel-Rammingen U, Janulczyk R, Collin M, et al. Low antibody levels against cell wall-attached proteins of Streptococcus pyogenes predispose for severe invasive disease. J Infect Dis. 2004;189:797-804.

19. Morefield G, Touhey G, Lu F, Dunham A, HogenEsch H. Development of a recombinant fusion protein vaccine formulation to protect against Streptococcus pyogenes. Vaccine. 2014;32:3810-5.

20. Morefield GL. A rational, systematic approach for the development of vaccine formulations. AAPS J. 2011;13(2):191-200. doi:10.1208/s12248-011-9261-1.

21. Glenny A, Pope C, Waddington H, Wallace U. The antigenic value of toxoid precipitated by potassium alum. J Pathol Bacteriol. 1926;29:38-45.

22. Glenny A, Buttle G, Stevens M. Rate of disappearance of diphtheria toxoid injected into rabbits and guinea pigs: toxoid precipitated with alum. J Pathol Bacteriol. 1931;34:267-75.

23. Clapp T, Siebert P, Chen D, Jones Braun L. Vaccines with aluminum-containing adjuvants: optimizing vaccine efficacy and thermal stability. J Pharm Sci. 2011;100:388-401.

24. HogenEsch H. Mechanism of immunopotentiation and safety of aluminum adjuvants. Front Immunol. 2013;3:406.

25. HogenEsch H, Dunham A, Burlet E, Lu F, Mosley YC, Morefield G. Preclinical safety study of a recombinant Streptococcus pyogenes vaccine formulated with aluminum adjuvant. J Appl Toxicol. 2017;37(2):222-30.

26. Roggiani M, Stoehr JA, Olmsted SB, Matsuka YV, Pillai S, Ohlendorf DH, et al. Toxoids of streptococcal pyrogenic exotoxin A are protective in rabbit models of streptococcal toxic shock syndrome. Infect Immun Sep. 2000;68(9):5011-7.

27. Silva-Costa C, Carriço JA, Ramirez M, Melo-Cristino J. Scarlet fever is caused by a limited number of Streptococcus pyogenes lineages and is associated with the exotoxin genes ssa, spe A and speC. Pediatr Infect Dis J. 2014;33(3):306-10.

28. Plainvert C, Doloy A, Loubinoux J, Lepoutre A, Collobert G, Touak $\mathrm{G}$, et al. Invasive group A streptococcal infections in adults, France (2006-2010). Clin Microbiol Infect. 2012;18(7):702-10. 